\title{
Le cure intermedie come soluzione per affrontare le malattie croniche: il programma Stroke Care
}

\section{Intermediate care as a solution for managing chronic diseases: The Stroke Care program in Emilia Romagna (Italy)}

\section{Simona Bartoli ${ }^{a, *}$, Salvatore Ferro ${ }^{b}$, Rossana De Palma ${ }^{a}$}

\author{
${ }^{a}$ Agenzia Sanitaria e Sociale Regionale, Regione Emilia Romagna \\ ${ }^{\mathrm{b}}$ Servizio Presidi Ospedalieri, Regione Emilia Romagna
}

Ricevuto il 28 marzo 2011; accettato l'11 luglio 2011

disponibile online il 12 ottobre 2011

\section{KEYWORDS}

Intermediate care;

Chronic diseases;

Stroke.

\begin{abstract}
Summary
Introduction: Chronic conditions represent an urgent health-care challenge for three main reasons: 1) their frequency is increasing all over the world, mainly as a result of longer life expectancy; 2) they require complex, long-term response that goes beyond the acute-care hospital setting; and 3) they place a heavy burden on health-care systems.

Materials and methods: The authors conducted a critical review of the literature to obtain an overview of the theoretical frameworks and strategies that have been developed to tackle chronic diseases, with special emphasis on intermediate care.

Results: Several frameworks and models of care delivery for people with long-term conditions were frequently discussed in the literature. Thus far, however, no consensus has been reached on the definition of "intermediate care". The authors report the experience of the Stroke Care program in the Emilia Romagna Region of Italy as an example of integrated care delivery. Discussion: The management of chronic diseases is considered a priority. Although there is no consensus definition of the term "intermediate care", the authors attempted to highlight its main characteristics. Their analysis of the Stroke Care program provides an illustration of how theory and practice are combined.

(c) 2011 Elsevier Srl. All rights reserved.
\end{abstract}

\footnotetext{
* Corrispondenza: Agenzia Sanitaria e Sociale Regionale, Area Governo Clinico, Regione Emilia Romagna, v.le A. Moro 21 - 40127 Bologna.
} E-mail: sbartoli@regione.emilia-romagna.it (S. Bartoli). 


\section{Il peso della cronicità}

Le malattie croniche rappresentano una delle più importanti sfide, in termini di gestione del paziente, che la medicina e l'assistenza sanitaria stanno affrontando e a cui dovranno far fronte nel prossimo futuro, come attestato dalle proiezioni di lungo periodo illustrate dalla World Health Organization (WHO) [1].

In tutto il mondo, tranne in Africa, la causa di morte più rilevante è costituita dalle malattie croniche e anche in Europa il tasso di mortalità per malattie croniche non si discosta significativamente da quello mondiale [2]. Tra i fattori determinanti l'insorgenza delle principali malattie croniche, oltre a elementi modificabili come dieta, attività fisica, stile di vita, è riconosciuto il ruolo preminente del progressivo allungamento dell'età della popolazione. La condizione dell'anziano, più complessa e influenzata dalla polipatologia, dalla concomitanza di trattamenti medici, dalla ridotta funzione di organi e apparati, richiede un approccio di cura personalizzato in grado di individuare l'intensità di trattamento del singolo paziente. Pertanto, è necessario adottare un modello assistenziale capace di rispondere a questi bisogni di cura.

Anche in Italia la gestione della cronicità costituisce un tema di assoluta rilevanza. Secondo le elaborazioni del Major and Chronic Diseases Report del 2007 [3], redatto dalla Direzione Generale Salute e Consumatori della Commissione Europea, gli italiani risultano più longevi rispetto alla media europea (Europa a 25) in quanto in Italia l'aspettativa di vita all'età di 65 anni è di circa 18 e 22 anni, rispettivamente per gli uomini e le donne. Poiché l'aumento della durata della vita si accompagna inevitabilmente a un deterioramento delle condizioni di salute, dopo i 65 anni la quota di anni di vita ad alto rischio d'insorgenza di patologie croniche diviene rilevante (50\% circa), seppur non differente in confronto ad altri Paesi europei.

Inoltre, la graduale contrazione del numero di posti letto delle strutture sanitarie avvenuta in Italia, la progressiva riduzione della degenza media dei ricoveri e la concentrazione dell'offerta nelle strutture ospedaliere per acuti hanno contribuito a rendere ancor più evidente l'esigenza di implementare soluzioni organizzative alternative che consentano di gestire appropriatamente il paziente cronico.

In generale, da tempo su questo tema si è concentrato l'interesse della comunità scientifica, impegnata a individuare strategie operative tese al miglioramento dell'assistenza al paziente affetto da malattie croniche. Per un utile inquadramento, è opportuno fare riferimento ad alcuni schemi concettuali che nel corso degli anni hanno contraddistinto l'approccio alla gestione della cronicità.

\section{La gestione dei malati cronici: dai modelli organizzativi alle strategie attuative}

Risale ai primi anni Novanta nei Paesi anglosassoni la proposta di un approccio strutturato alla cura del paziente cronico con la formulazione del modello organizzativo della "continuity of care" [4]. Successivamente diverse esperienze hanno avuto alterna fortuna [5,6] e si è affermato come modello di riferimento il Chronic Care Model (CCM) disegnato nel 2001 dal professor Wagner, direttore del MacColl
Institute for Healthcare Innovation. In questo schema, all'interno di un framework basato su sei elementi essenziali (comunità, organizzazioni sanitarie, supporto all'autocura, modelli di erogazione dell'assistenza, supporto evidencebased alle decisioni e sistemi informativi) vengono enfatizzati alcuni elementi fondamentali: l'approccio di sistema, l'integrazione professionale e i programmi di prevenzione [7]. Il CCM è caratterizzato dalla proattività delle relazioni che coinvolge non solo medico e paziente, ma anche la famiglia, la comunità di riferimento e tutti quei servizi di assistenza sociosanitaria di cui il paziente può usufruire. In tal senso, l'adozione del CCM come schema di riferimento richiede l'avvio di un processo di cambiamento culturale e organizzativo che considera, in primo luogo, la capacità dei servizi e dei professionisti di erogare risposte integrate fra loro e orientate a porre il paziente al centro del progetto assistenziale. Il CCM si pone così come risposta complessiva alla necessità di superare il tradizionale modello di organizzazione sanitaria, basato sul trattamento rapido ed efficiente delle patologie acute.

Nel 2002 il WHO ha sviluppato in misura maggiore gli aspetti strategici e di comunità del CCM, formalizzandone la nuova versione denominata "Innovative Care for Chronic Conditions" (ICCC) [8]. Questo modello, che nel concetto di "condizioni croniche" include - oltre alle tradizionali malattie croniche o non trasmissibili - anche condizioni specifiche come disordini mentali e deficit transitori [8], individua l'esistenza di tre livelli assistenziali in grado di influenzare la cura dei pazienti cronici: il livello macro, espresso dalle scelte politiche, di regolamentazione e finanziamento dell'assistenza; il livello meso, rappresentato dalle comunità e organizzazioni sanitarie; il livello micro, costituito dal paziente e dalla sua famiglia. Il modello ICCC s'incardina sul rapporto di collaborazione tra paziente, team multidisciplinare di professionisti e comunità territoriale che si configura, quindi, come l'elemento determinante per il raggiungimento di outcome positivi: infatti, il paziente cronico non esaurisce il proprio bisogno di cura all'interno di una struttura sanitaria, ma necessita di assistenza o monitoraggio continui fuori dall'ospedale. Inoltre, i livelli individuati compongono una triade che può agire efficacemente se inserita in un ambiente favorevole al suo sviluppo, ossia in un contesto, il livello macro, in cui le scelte strategiche, i principi e il governo del sistema siano coerenti e sostengano la cura del paziente cronico secondo la logica dell'integrazione [8]. Al di là dell'evoluzione nella struttura del modello, con l'esperienza dell'ICCC si vuole concentrare l'attenzione dei sistemi sanitari su alcuni temi strategici, come il coordinamento dell'assistenza in termini organizzativi, l'introduzione del case-manager, l'utilizzo dei sistemi informativi e la prevenzione.

Questi elementi introducono in modo chiaro e diretto il tema complesso delle modalità più efficaci per l'erogazione dell'assistenza ai malati cronici. Tra gli approcci più conosciuti vi è il modello del "Kaiser triangle", sviluppato sulla base del CCM dal Kaiser Permanente negli Stati Uniti [9]. II modello, pensato e organizzato secondo un approccio di "popolazione", individua nella "piramide dei rischi" lo strumento, ormai diffuso ${ }^{1}$, utile a stratificare la popolazione

\footnotetext{
${ }^{1}$ Si pensi a titolo di esempio all'impostazione del "NHS and social care model" del 2005 nel Regno Unito.
} 
affetta da malattie croniche a seconda del rischio clinico, e a indirizzare le modalità di cura in modo adeguato a ogni profilo di rischio. In particolare, alla base della piramide il primo livello, in cui si identifica la malattia cronica con basso rischio di complicanze, corrisponde all'autocura; al secondo livello (rischio moderato) si collega l'attività di un team multidisciplinare responsabile del disease management; al terzo livello, corrispondente alla fascia più grave di rischio, si associa un'elevata intensità di cura con la presa in carico da parte del case-manager. In sintonia col CCM, anche il triangolo di Kaiser è orientato al raggiungimento dei medesimi obiettivi in termini di rafforzamento del ruolo del paziente e consolidamento di team integrati multiprofessionali.

Se, quindi, appaiono chiari i modelli di riferimento, non altrettanto lo sono le strategie necessarie ai policymaker per realizzare un'adeguata implementazione dei modelli proposti. Secondo Busse et al. [10] le modalità per affrontare il tema della gestione delle patologie croniche possono essere ricondotte ai temi della prevenzione, dell'innovazione nell'organizzazione assistenziale, dei programmi di disease management e dei modelli di cura integrata, interventi che oggi, indiscutibilmente, richiedono di essere corredati dalla valutazione dell'impatto clinico ed economico. È risaputo, infatti, che l'associazione di interventi diversi nell'ambito della prevenzione e della diagnosi precoce di malattia - come dimostrato per iniziative relative a ipertensione, diabete e fumo - si rivela maggiormente efficace, ma connotata da un incremento di spesa che necessita di attenta considerazione in termini di costoopportunità.

Per quanto riguarda le innovazioni organizzative, oggi l'interesse si divide tra le sfide associate all'emergere di nuove competenze e responsabilità professionali e quelle legate all'individuazione di nuovi setting assistenziali. Alcuni Paesi europei - come Regno Unito, Paesi Bassi e Germania stanno promuovendo la figura professionale, denominata "nurse practitioner" o "community nurse", nella quale la professionalità dell'infermiere tradizionale si arricchisce di compiti usualmente attribuiti al medico, quali la prescrizione di alcuni farmaci o l'erogazione di taluni interventi meno complessi. Anche in Italia sempre maggiore attenzione è rivolta alla figura del "case-manager" come colui che valuta i bisogni di cura del paziente, pianifica le azioni, favorisce l'accesso alle cure appropriate, ne monitora la qualità e mantiene le relazioni tra le strutture sanitarie, il paziente e la sua famiglia. Nell'ambito dei nuovi setting organizzativi, l'orientamento dei diversi sistemi sanitari predilige i modelli incentrati sulla collaborazione tra professionisti, come le pratiche di gruppo o i "nurse-led clinics", che permettono di valorizzare e rafforzare il ruolo delle cure primarie e offrono una risposta qualificante per esiti clinici e soddisfazione del paziente, anche se non sempre economicamente vantaggiosa. Infine, per molti aspetti simili, i programmi di disease management così come i modelli di assistenza integrata, ormai ampiamente diffusi in Europa, sono riconosciuti efficaci per il miglioramento della qualità dell'assistenza [11].

In sintesi, nonostante le numerose esperienze avviate in questi anni si siano caratterizzate per specifici aspetti, in tutte è possibile rintracciare un "file rouge" costituito da alcuni elementi comuni, tra cui l'individuazione di percorsi assistenziali evidence-based, l'utilizzo di sistemi informativi a supporto delle decisioni, il coordinamento delle cure per il raggiungimento della continuità assistenziale, l'integrazione dei professionisti. Tuttavia, ancora molto può e deve essere fatto in merito alla valutazione dell'impatto di tali strategie nei sistemi sanitari che, a tutt'oggi, raramente si configurano come conclusive in termini sia di reali benefici conseguiti sia di confronto con altre modalità di intervento.

\section{Un modello di assistenza integrata in Emilia Romagna: le cure intermedie e il programma Stroke Care}

Le cure intermedie rientrano nell'ultimo gruppo di strategie sopra esaminate, in quanto rappresentano una modalità di coordinamento e integrazione dell'assistenza indirizzata a una pluralità di malattie croniche, al fine di assicurare la continuità delle cure qualora non si sia verificato il completo recupero dell'autonomia o non vi siano le condizioni per il trattamento ambulatoriale, domiciliare o residenziale.

A tutt'oggi la comunità internazionale non ha ancora raggiunto una definizione univoca di "cure intermedie" [12] così da esser considerate talvolta sinonimo delle cosiddette "cure di transizione" [13] e talaltra distinte, in quanto le prime si connotano come soluzioni strutturali a differenza delle seconde che sono invece indicative del processo assistenziale [14].

Nei Paesi anglosassoni in cui la cultura delle cure territoriali è avanzata, la British Geriatrics Society ne sottolinea, quali aspetti peculiari, la valutazione multifunzionale, la convalescenza, la riabilitazione e l'erogazione di cure temporanee [15]. Il National Health Service, nel National Service Framework for Older People, le definisce come un livello di cura interposto tra cure primarie e specialistiche e individua, come caratteristica essenziale, l'essere integrate in un unitario sistema di assistenza con le cure primarie e secondarie e con l'assistenza sociale, all'interno di una partnership tra soggetti pubblici e privati [16]. Anche ultimamente l'Intermediate Care Learning Network scozzese, nel confermare la precedente definizione, pone l'attenzione sulla comune finalità dei servizi che, per quanto diversi e con differenti operatività, sono tutti finalizzati a mantenere il paziente nella propria abitazione quanto più possibile [17]. Nello specifico, obiettivi qualificanti sono rappresentati dal favorire il passaggio del paziente dall'ospedale a casa e viceversa accelerando la ripresa dalla malattia, prevenendo ricoveri ospedalieri non necessari, facilitando le dimissioni anticipate, massimizzando l'autonomia dei pazienti.

Nel contesto italiano è col Piano Sanitario Nazionale 20032005 che si individua nel territorio la primaria sede di assistenza e governo dei percorsi sanitari e sociosanitari e si ravvisa la necessità di sviluppare una rete integrata di servizi sanitari e sociali per l'assistenza ai malati cronici, agli anziani e ai disabili, senza mai esplicitamente richiamare le cure intermedie. Nello stesso periodo, si contraddistingue l'esperienza toscana che impegna attenzione e risorse nello sviluppo delle cure domiciliari e residenziali per pazienti di tipo complesso. È del 2003 la definizione formulata da Comodo e Maciocco [18] secondo i quali "le cure intermedie sono un'area di servizi integrati, sanitari e sociali (domiciliari, residenziali e semiresidenziali) finalizzata a garantire la 
continuità assistenziale dopo la dimissione ospedaliera e a favorire il rapido recupero funzionale e la massima autonomia dei pazienti". Più recentemente alcuni autori hanno cercato di sistematizzare il concetto di cure intermedie riportando le condizioni che devono essere soddisfatte secondo la Intermediate Care: Guidance for commissioners and providers of health and social care sottoposta a revisione l'ultima volta nel 2010 dalla British Geriatrics Society [19] e prevedendone alcune modalità specifiche. Un servizio di assistenza appartiene alle cure intermedie se è basato su un approccio globale al paziente, se si avvale di un team multidisciplinare che condivide la valutazione del caso, se è finalizzato al recupero funzionale del paziente e al rientro al domicilio, se viene erogato al massimo per sei settimane, se il paziente, in assenza di tale servizio, necessiterebbe di un'ospedalizzazione di lungo periodo o di assistenza residenziale. Per quanto riguarda le forme di cure intermedie più diffuse, invece, gli autori identificano l'ospedalizzazione a domicilio, la lungodegenza, il day-hospital, la residenza sanitaria assistenziale, l'ospedale di comunità.

A livello legislativo, il documento preliminare del Piano Sanitario Nazionale 2010-2012 suggerisce, tra le azioni per lo sviluppo del sistema, la promozione della "continuità dell'assistenza e la gestione integrata delle cronicità tra territorio e ospedale" con riferimento alle "strutture di cure intermedie".

In sintesi, nonostante la molteplicità di definizioni e articolazioni organizzative che in questi anni sono state proposte nel mondo e in Italia, è possibile rintracciare la condivisione di alcuni elementi comuni come i destinatari e le finalità. I primi sono rappresentati da: a) pazienti che, superata la fase acuta della malattia, risultano stabilizzati clinicamente, ma necessitano ancora di una fase di osservazione e continuità terapeutica e riabilitativa; b) pazienti per $i$ quali vi siano le premesse per un recupero funzionale; c) pazienti che necessitano di assistenza sanitaria, ma non ad alto contenuto tecnologico e ad alta intensità assistenziale. Gli obiettivi sono: a) garantire una continuità di cure ospedale-territorio e all'interno delle reti dei servizi ospedalieri; b) ridurre le riammissioni ospedaliere; c) riportare il paziente al domicilio o in strutture territoriali.

In regione Emilia Romagna l'attenzione alla problematica si consolida dagli anni Novanta con alcuni atti legislativi mirati prevalentemente a garantire l'assistenza agli anziani disabili (LR 5/1994, Tutela e valorizzazione delle persone anziane - interventi a favore di anziani non autosufficienti; DGR 124/1999, Criteri per la riorganizzazione delle cure domiciliari; DGR 1377/1999, Criteri, modalità e procedure per la contribuzione alle famiglie disponibili a mantenere l'anziano non autosufficiente nel proprio contesto, DGR $1378 / 1999$, Integrazione delle prestazioni sociali, sanitarie e a rilievo sanitario, a favore di anziani non autosufficienti assistiti nei servizi integrati sociosanitari; DGR 2068/2004, Il sistema integrato di interventi sanitari e socio-assistenziali per persone con gravissime disabilità acquisite in età adulta. Prime indicazioni), fino al Piano Sanitario e Sociale Regionale 2008-2010 che riconosce come area prioritaria d'intervento i modelli organizzativi che favoriscano l'integrazione fra assistenza in regime di acuzie, assistenza postacuta e riabilitazione. In questa logica l'area delle "cure intermedie" assume particolare rilevanza organizzativa e gestionale perché in grado di assicurare la continuità assistenziale attraverso la ridefinizione di ruoli e responsabilità delle professioni (particolare del personale infermieristico) e l'esplicitazione di funzioni e ruoli delle sedi di cura, articolate per intensità, in rapporto al territorio di riferimento.

Il programma Stroke Care avviato in Emilia Romagna nel 2006 offre un esempio concreto di come può essere realizzata l'assistenza integrata al paziente con ictus attraverso lo sviluppo delle cure intermedie [20]. È ormai riconosciuto che l'assistenza in aree di degenza dedicate (Stroke Unit) con una precoce e completa presa in carico da parte di un team multidisciplinare di operatori esperti, un rapido accesso alla diagnostica per immagini, un precoce avvio dell'intervento riabilitativo e della dimissione protetta migliorano la sopravvivenza e diminuiscono il rischio di disabilità del paziente colpito da ictus.

In Emilia Romagna l'interesse alla problematica risale alla fine degli anni Novanta quando si afferma, per il trattamento delle patologie cerebrovascolari, la necessità di trasformare le cure domiciliari in un modello di assistenza domiciliare integrata, superando la tradizionale frammentarietà degli interventi sul territorio (DGR 124/1999). Successivamente, una volta affermato il modello organizzativo regionale "hub \& spoke" in attuazione del Piano Sanitario Regionale 19992001, l'attenzione si concentra sulla definizione di un percorso integrato di prevenzione, cura e riabilitazione che tenga conto della complessità del caso trattato e, di conseguenza, sia in grado di modulare l'assistenza a seconda dell'intensità di cura richiesta. Le reti di emergenza-urgenza e la rete socioassistenziale sono state anch'esse sviluppate per offrire una risposta integrata di cura dal momento del riconoscimento del paziente con ictus a quello della dimissione protetta.

Nel 2007, col Documento di indirizzo per l'organizzazione dell'assistenza integrata al paziente con ictus Programma Stroke Care (DGR 1720/2007), vengono definite le linee di indirizzo per l'organizzazione dell'assistenza integrata al paziente con ictus basate sull'integrazione interdisciplinare e interprofessionale durante l'intero percorso di cura (fase preospedaliera, ospedaliera, postospedaliera). La continuità assistenziale è altresì garantita dal potenziamento del ruolo dell'infermiere come responsabile del caso sia durante il ricovero (case-manager ospedaliero) sia dopo la dimissione (case-manager territoriale). In particolare, la collaborazione e il coordinamento delle attività tra i due case-manager favorisce l'integrazione tra assistenza ospedaliera, territoriale e sociale. Nelle linee di indirizzo si delinea anche l'organizzazione della rete regionale di assistenza al paziente con ictus, che costituisce un raccordo funzionale tra il sistema di emergenza territoriale, quello di assistenza ospedaliera e quello sociosanitario nella gestione postacuta. Ogni Azienda Sanitaria può modellare la propria "rete stroke" su scala geografica con diversa estensione territoriale (Area Vasta, Provincia, Distretto), a seconda delle esigenze organizzative locali. Il funzionamento della "rete stroke" deve essere assicurato da protocolli operativi condivisi che in fase acuta permettano un rapido inquadramento diagnostico, finalizzato al trasferimento del paziente nelle strutture ospedaliere più idonee (in relazione alla tipologia di interventi da erogare) del territorio provinciale, e che nella fase postospedaliera favoriscano percorsi assistenziali integrati con i servizi extraospedalieri territoriali di riferimento. 
In particolare, per la gestione postacuta si prevedono tre percorsi assistenziali differenziati in base alla gravità e alla persistenza nel tempo degli esiti dell'ictus. Il percorso "follow up", quello di "riabilitazione territoriale" e il "progetto personalizzato a lungo termine" si caratterizzano per un gradiente crescente di intensità dell'assistenza richiesta e di integrazione con i servizi sociosanitari. Se il secondo tipo di percorso favorisce la dimissione precoce, il terzo tipo, coinvolgendo persone non autosufficienti, necessita invece dell'implementazione di dimissioni protette. Pertanto, a seconda delle conseguenze dell'ictus, la continuità dell'assistenza durante la fase postospedaliera può essere garantita nei diversi setting assistenziali come per esempio quello domiciliare, riabilitativo territoriale oppure residenziale.

Questi interventi normativi, integrati recentemente dalla DGR 742/2010 che istituisce la Commissione tecnico-scientifica regionale "Programma Stroke Care", configurano il quadro complessivo di riferimento per l'assistenza al paziente colpito da ictus. Se la fase di promozione delle forme di coordinamento e integrazione dell'assistenza può considerarsi in via di completamento, l'implementazione delle attività e la predisposizione dei servizi finalizzati a garantire la continuità assistenziale è ad oggi ancora in corso. Pertanto, su ispirazione di quanto è già avvenuto a livello internazionale riguardo alle diverse modalità di gestione delle malattie croniche, nei prossimi anni occorrerà avviare una riflessione sia sulla rispondenza del modello di assistenza adottato alle aspettative iniziali sia sulla sua efficacia. Entrambe le valutazioni possono essere sviluppate, come suggerito dalla letteratura esaminata, elaborando indicatori in grado di misurare l'efficienza nell'utilizzo di risorse, la funzionalità del processo di cura e il suo impatto organizzativo, nonché l'esito clinico associato.

\section{Conflitto di interessi}

Gli autori dichiarano di essere esenti da conflitto di interessi.

\section{Bibliografia}

[1] World Health Organization. The Global Burden of Disease: 2004 update. Geneva: WHO Press; 2008.

[2] World Health Organization. Preventing Chronic Diseases: A vital investment. Geneva: WHO Press; 2005.
[3] Task Force on Major and Chronic Diseases of DG SANCO's Health Information Strand. Major and Chronic Diseases Report 2007. Luxembourg: European Communities, 2008.

[4] Robles SC. A public health framework for chronic disease prevention and control. Food Nutr Bull 2004;25(2):194-9.

[5] Suñol R, Carbonell JM, Nualart L, Colomés L, Guix J, Bañeres J, et al. Towards health care integration: the proposal of an evidence- and management system-based model. Med Clin (Barc) 1999;112(Suppl 1):97-105.

[6] Health Services Management Centre-University of Birmingham, NHS Institute for Innovation and Improvement. Improving care for people with long-term conditions: A review of UK and international framework. Birmingham, 2006.

[7] Wagner EH, Austin BT, Davis C, Hindmarsh M, Schaefer J, Bonomi A. Improving chronic illness care: translating evidence into action. Health Aff (Millwood) 2001;20(6):64-78.

[8] World Health Organization. Innovative Care for Chronic Conditions: Building blocks for action. Noncommunicable diseases and mental Health. Geneva: WHO Press; 2002.

[9] Feachem RG, Sekhri NK, White KL. Getting more for their dollar: a comparison of the NHS with California's Kaiser Permanente. BMJ 2002;324(7330):135-41.

[10] Busse R, Blümel M, Scheller-Kreinsen D, Zentner A. European Observatory on Health Systems and Policies. Tackling Chronic Disease in Europe. Strategies, interventions and challenges. Observatory Studies Series No 20. WHO, 2010.

[11] Velasco-Garrido M, Busse R, Hisashige A. Are Disease Management Programmes (DMPs) Effective in Improving Quality of Care for People with Chronic Conditions? Copenhagen: WHO Regional Office for Europe; 2003.

[12] Melis RJ, Olde Rikkert MG, Parker SG, van Eijken MI. What is intermediate care? BMJ 2004;329(7462):360-1.

[13] Coleman EA. Falling through the cracks: challenges and opportunities for improving transitional care for persons with continuous complex care needs. J Am Geriatr Soc 2003;51 (4):549-55.

[14] Compagni A, Tediosi F, Tozzi V. L'integrazione tra ospedale e territorio nelle Aziende Sanitarie. In: Anessi Pessina E, Cantù E (a cura di). Rapporto OASI 2010. L'aziendalizzazione della sanità in Italia. Milano: Egea, 2011.

[15] British Geriatrics Society. Intermediate Care: Guidance for commissioners and providers of health and social care. Best Practice Guide 4.2. 2008.

[16] Department of Health. National Service Framework for Older People. London: Department of Health; 2001.

[17] Intermediate Care Learning Network. Intermediate care. Agreeing a common definition. 2011.

[18] Comodo N, Maciocco G. Cure intermedie: basi concettuali. Prospettive Sociali Sanitarie 2004;3:1-3.

[19] Salsi A, Calogero P. Le cure intermedie. It J Med 2010;4(4):57-62.

[20] Regione Emilia-Romagna. Assistenza all'ictus. Modelli organizzativi regionali. Dossier 166. Bologna, 2008. 